\title{
El desarrollo de Habilidades Docentes y Asesoría Académica del alumnado de escuelas normales públicas mexicanas en colegios de Murcia España: una experiencia exitosa
}

\author{
The development of teaching school skills and academic advising of students \\ of normal public Mexican schools in colleges of Murcia Spain: A successful \\ experience
}

\section{O desenvolvimento de Habilidades de Ensino e Aconselhamento Acadêmico de estudantes de escolas públicas públicas mexicanas em escolas em Murcia} Espanha: uma experiência bem sucedida

Román Castro Miranda Benemérita Normal Veracruzana "Enrique C. Rébsamen”, México romancastrobenv@gmail.com https://orcid.org/0000-0002-8905-7286

\section{Resumen}

El presente artículo describe una experiencia exitosa sobre el desarrollo de habilidades docentes y la asesoría académica del alumnado de escuelas normales públicas de 15 estados de la república mexicana, donde se realizaron prácticas profesionales en colegios de educación infantil, primaria y secundaria de Murcia, España, con el objetivo de analizar aspectos formativos de dicho alumnado en situaciones reales de trabajo. La metodología empleada es de investigación-acción, ya que se pretende reflexionar sobre cómo resolver los problemas cotidianos e inmediatos de sus prácticas profesionales concretas, innovar e incorporar desde sus referentes, acciones y estrategias que les ayudarán en la toma de decisiones sobre sus propias problemáticas detectadas y realizar adecuaciones a las planeaciones didácticas que tenían como encomienda. Resulta interesante identificar algunas reflexiones en torno a cómo el estudiantado que proviene de distintos puntos del territorio mexicano cuentan con referentes, licenciaturas y contextos proporcionalmente distintos a los que se involucrarían; sin embargo, la perspectiva metodológica, el manejo de estrategias didácticas y algunas habilidades docentes, tales como el manejo de grupo, la interacción entre ellos y su alumnado, permitió que se insertaran 


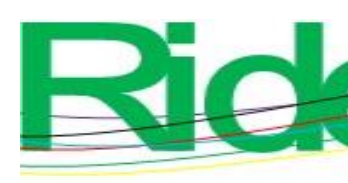

Revista Iberoamericana para la

Investigación y el Desarrollo Educativo

ISSN $2007-7467$

significativamente en la vida docente y dinámica escolar de los colegios donde fueron asignados.

Palabras clave: asesoría académica, estrategias didácticas, habilidades docentes, participación colegiada, práctica profesional.

\section{Abstract}

This article describes a successful of development of teaching school skills and academic advising for students from public normal schools in 15 states of the Mexican Republic, who completed professional internships at schools for early childhood, primary and secondary education in Murcia, Spain with the objective of analyzing training aspects of said students in real work situations. The methodology used is research-action, as through this is intended to reflect on how to solve the daily and immediate problems of their specific professional practices, innovate and incorporate from their referents actions and strategies that will help them in making decisions about their own problems detected and make adjustments to the didactic planning they had as commission. It is interesting to identify some reflections on how the student body comes from different points of the Mexican territory; having references, degrees and contexts proportionally different from those that would be involved; however, the methodological perspective, the management of didactic strategies and some teaching skills, such as group management, the interaction between them and their students, allowed them to be inserted significantly into the teaching life and school dynamics of the schools where they were assigned.

Keywords: academic advice, teaching strategies, teaching skills, collegiate participation, professional practice.

\section{Resumo}

Este artigo descreve uma experiência bem-sucedida sobre o desenvolvimento de habilidades pedagógicas e o aconselhamento acadêmico de estudantes de escolas públicas públicas em 15 estados da República Mexicana, onde foram realizadas práticas profissionais em escolas para a infância, educação primária e secundária em Múrcia, Espanha. com o objetivo de analisar os aspectos de treinamento dos alunos em situações de trabalho real. A metodologia utilizada é a pesquisa-ação, pois visa refletir sobre como resolver os problemas diários e imediatos de suas práticas profissionais específicas, inovar e incorporar seus referentes, ações e estratégias que os 


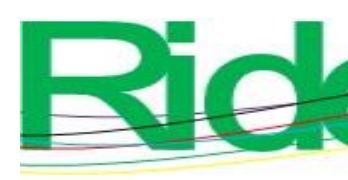

Revista Iberoamericana para la

Investigación y el Desarrollo Educativo

ISSN 2007 - 7467

ajudarão a tomar decisões sobre seus próprios problemas detectou e fez ajustes aos planos didáticos que foram confiados. É interessante identificar algumas reflexões sobre como o corpo estudantil que vem de diferentes pontos do território mexicano tem referências, graus e contextos proporcionalmente diferentes dos que estarão envolvidos; No entanto, a perspectiva metodológica, o gerenciamento de estratégias didáticas e algumas habilidades de ensino, como o gerenciamento grupal, a interação entre eles e seus alunos, permitiram que fossem inseridos significativamente na vida docente e na dinâmica escolar das escolas onde foram designadas.

Palavras-chave: aconselhamento acadêmico, estratégias didáticas, habilidades de ensino, participação colegiada, prática profissional.

Fecha Recepción: Abril 2017 Fecha Aceptación: Septiembre 2017

\section{Introducción}

Los estudiantes del nivel superior encargados de llevar el conocimiento a diferentes instituciones de educación (básica, media superior o superior) se encuentran con ciertos retos para llevar a cabo su trabajo. Vivir en el presente siglo significa trabajar en un contexto internacional de globalización de los conocimientos y habilidades docentes, con todos sus desafíos y oportunidades, donde la educación no solo cumple un papel estratégico en el crecimiento económico, sino también en la configuración de un contexto socialmente diverso. En este sentido, las escuelas Normales Públicas del país se encuentran favoreciendo prácticas profesionales para que el estudiantado aprenda la labor profesional a través de la práctica. Es así que realizar estancias académicas contribuye con el papel de formar docentes en contextos diferenciados. La importancia que reviste esta experiencia se debe a que, durante esta estancia, el maestro en formación pone en práctica los saberes teóricos, heurísticos y axiológicos.

El presente artículo pretende describir los resultados obtenidos durante la estancia académica realizada en Murcia, España, por alumnos de octavo semestre de escuelas normales públicas de 15 estados de la república mexicana, con el objetivo de analizar aspectos formativos de dicho alumnado (quienes se encontraban cursando especialidades en matemáticas, inglés, telesecundaria y español) en situaciones reales de trabajo en cinco colegios de educación infantil (preescolar), primaria, especial, educación física y educación secundaria. 


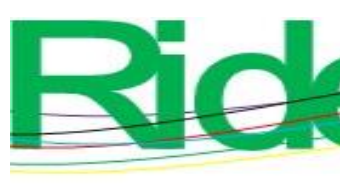

Revista Iberoamericana para la

Investigación y el Desarrollo Educativo

ISSN $2007-7467$

El programa de participación se desprende de una convocatoria emitida por la Dirección General de Educación Superior para Profesionales de la Educación (DGESPE), a través de la Coordinación Nacional de Becas para Educación Superior (CNBES), que dan oportunidad a esta estancia académica a 96 alumnos de casi 600 aspirantes y 6 catedráticos de 100 inscritos, en convenio entre el gobierno español y el mexicano, que designan tres sedes para realizar estas prácticas profesionales. Cabe destacar que una estancia académica profesional es aquella actividad realizada durante el desarrollo de un proyecto a través de la participación directa del estudiante en el desempeño de su propia profesión, con el fin de poder contribuir a la formación integral del estudiante, a través de la combinación de conocimientos teóricos adquiridos en el aula con aspectos prácticos de la realidad laboral y profesional.

Los participantes fueron situados en tres grupos: el primero conformado por 32 alumnos y dos catedráticos en Cuenca, provincia ubicada al centro de España en la región de CastillaLa Mancha; el segundo grupo se estableció con 31 alumnos y dos catedráticos en Málaga, sexta ciudad más poblada de España y perteneciente a la región de Andalucía, y en la tercera sede conformado por 33 alumnos y dos catedráticos en Murcia, ubicada al sudoeste de España y a 441 kilómetros de Madrid.

Cabe destacar, en palabras de Pelayo (2012), que durante una estancia académica se contemplan múltiples propósitos que van desde la inserción en colegios y escuelas secundarias españolas, hasta el favorecimiento de competencias docentes que implicaron el manejo de la planeación didáctica en las aulas, cuyo fin es contribuir en esta labor de los profesores dedicados a la educación y que empezarán a trabajar como tal, y con esta experiencia el objetivo principal de investigación queda enmarcada en la reflexión y análisis de las prácticas profesionales realizadas de abril a junio de 2015, por parte del alumnado que trabajó en Murcia, España, y enfocadas a diseñar un plan de actuación reflexiva, ponerlo en práctica y evaluarlo, considerando aspectos contextuales de cada zona escolar y de aula, así como todos aquellos elementos que favorecieran el aprendizaje significativo de su alumnado.

Habrá que señalar que, respecto a la estancia académica en lo general, algunas acciones se fueron redefiniendo por la propia naturaleza del programa de tutoría y seguimiento de la estancia, ya que en colegiado con catedráticos y tutores de la facultad de educación de la Universidad de Murcia se adicionaron actividades de tutoría, acompañamiento de su práctica profesional y capacitación de los aspectos sustantivos de los propios planes y programas de estudio de cada nivel educativo en el que se incorporó al estudiantado, dependiendo del nivel o modalidad del servicio educativo en el que se encontraban estudiando en México. 
Tabla 1. Procedencia de los participantes en la sede de Murcia, España

\begin{tabular}{|c|c|c|}
\hline Estado & Alumnado & Catedráticos \\
\hline Baja California & 4 & \\
\hline Baja California Sur & 3 & 1 \\
\hline Chihuahua & 2 & \\
\hline Durango & 2 & \\
\hline Guanajuato & 1 & \\
\hline Guerrero & 1 & \\
\hline Jalisco & 3 & \\
\hline Nuevo León & 2 & \\
\hline Puebla & 1 & \\
\hline San Luis Potosí & 2 & \\
\hline Sinaloa & 4 & \\
\hline Tabasco & 1 & \\
\hline Tamaulipas & 4 & \\
\hline Tlaxcala & 1 & \\
\hline Veracruz & 2 & 1 \\
\hline Subtotal & 33 & 2 \\
\hline Total & & 35 \\
\hline
\end{tabular}

Fuente: autoría propia.

De manera natural, el alumnado y los catedráticos se incorporaron a actividades académicas de la propia Universidad de Murcia, puesto que, desde su organización, las prácticas escolares están a cargo de la facultad de educación de la universidad, quienes de inicio informaron sobre la estructura y funcionamiento del sistema educativo español y sobre la organización de los contenidos y competencias de cada nivel y licenciatura según cada especialidad.

Durante el arribo a la región de Murcia, se dio inicio a la elaboración de un programa de capacitación que diversificara las actividades de práctica profesional, al tiempo que el estudiantado era asignado a cada colegio y empezaba a conocer el contexto socioeducativo y el desarrollo de las clases de sus tutores en cada aula. 


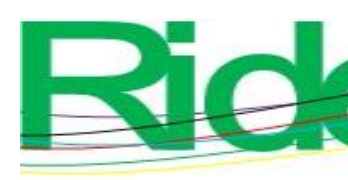

Revista Iberoamericana para la

Investigación y el Desarrollo Educativo

ISSN 2007 - 7467

El horario del alumnado durante sus prácticas fue el mismo que el de un maestro del colegio español, incluyendo el recreo y las reuniones con docentes y equipos directivos que se consideraran convenientes. También participaron en las actividades extraescolares que organizó cada colegio.

Con el fin de explicar con mayor precisión en las siguientes líneas expondré algunos temas de interés que dan sustento a la investigación, para así obtener una formación adecuada del profesor.

\section{Participación colegiada}

El trabajo en Murcia, España, se desarrolló bajo una propuesta de investigación-acción, trabajo muy desarrollado hoy en el ámbito educativo, ya que permite aprovechar recursos bastos en el quehacer dentro y fuera del aula. Este medio permite generar, en palabras de ÁlvarezGayou (2003), procedimientos de investigación centrados en la búsqueda de mejores resultados, ayudados por la participación de los actores, que al mismo tiempo aprenden y se desarrollan como personas.

Algunos aspectos característicos que este programa consideró, dentro de la práctica profesional del alumnado de octavo semestre de las diferentes licenciaturas y especialidades participantes, consistió básicamente en dar continuidad al proceso formativo que tanto ellos como los catedráticos que tenían como referentes (Secretaría de Educación Pública, 1997, 1999 y 2012). Cabe destacar que quienes se involucraron en este seguimiento fungieron como asesores académicos de séptimo y octavo semestres en escuelas normales públicas mexicanas, al igual que el estudiantado, quienes cursaban los mismos semestres, por lo que incorporar aspectos como el desarrollo de habilidades docentes, tutoría y prácticas profesionales, representaba un terreno conocido y definido para los catedráticos, al menos en el ámbito y campo de acción.

El trabajo pretendió guiar al estudiantado durante las visitas a los colegios asignados, sin embargo, también se les permitió innovar e incorporar desde sus referentes, acciones y estrategias que les ayudaran en la toma de decisiones sobre sus propias problemáticas detectadas y realizar adecuaciones a las planeaciones didácticas que tenían como encomienda, ya que diseñar, aplicar y evaluar la participación de 33 estudiantes de escuelas normales de 15 estados de la república mexicana en colegios de Murcia no era tarea sencilla.

La figura 1 pretende ilustrar el proceso de trabajo que permitió estructurar de abril a junio de 2015 el diseño de participación en los colegios de Murcia. 
Figura 1. Diseño básico de la investigación-acción en los colegios de Murcia, España

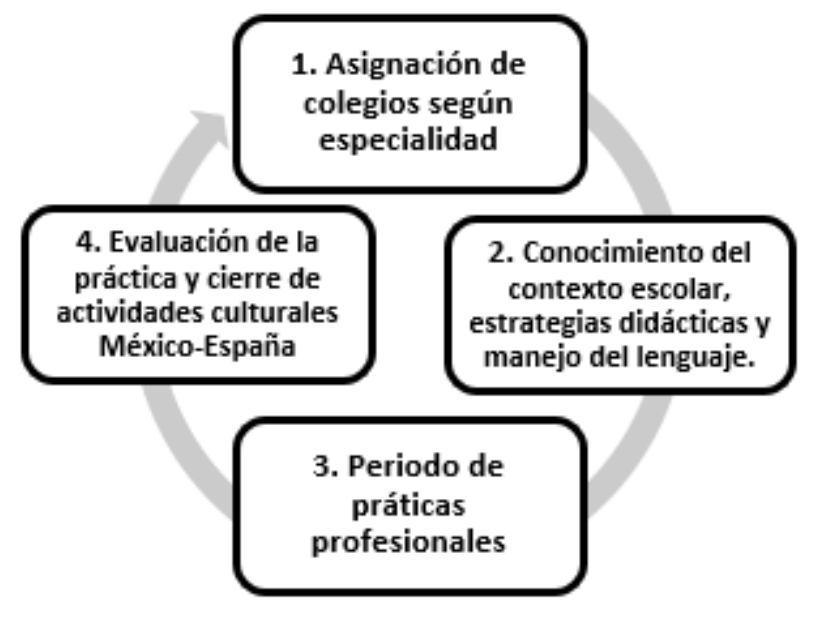

Fuente: autoría propia.

La perspectiva que se destaca pretende esencialmente "propiciar el cambio social, transformar la realidad y que las personas tomen conciencia de su papel en el proceso de transformación" (Sandín, 2003, p. 161).

El estudiantado provenía de distintos puntos del territorio mexicano, por lo que sus referentes, licenciaturas y contextos eran proporcionalmente distintos a los que se involucrarían, por ello, la perspectiva metodológica elegida favorece y se inserta en lo que se considera una postura predominantemente pragmática de la vida docente cotidiana, donde se pretende buscar soluciones inmediatas.

La perspectiva de investigación-acción se apoya en la metodología técnico-científica, ya que, como lo menciona Lewin (1946), se definió a la investigación-acción como una forma de cuestionamiento autorreflexivo, llevada a cabo por los propios participantes, en determinadas ocasiones con la finalidad de mejorar la racionalidad y la justicia de situaciones de la propia práctica social educativa, con el objetivo también de mejorar el conocimiento de dicha práctica y sobre las situaciones en las que la acción se lleva a cabo.

En palabras de Moser (1978), "el conocimiento práctico no es el objetivo de la investigación acción sino el comienzo", es decir, aunque el contexto español y las prácticas en colegios españoles era un escenario muy distinto para ellos, su experiencia en aulas mexicanas permitió guiar el rumbo de sus nuevas acciones en esta experiencia pedagógica. 


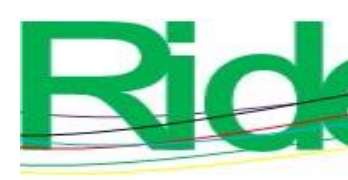

Revista Iberoamericana para la

Investigación y el Desarrollo Educativo

ISSN 2007 - 7467

La parte fundamental era entender la enseñanza y reflexionar sobre ella, y que el alumnado de octavo semestre, como participantes de este proceso, eran prácticamente considerados como docentes, pues potencialmente estaban a unos cuantos días de egresar de sus escuelas formadoras en México.

\section{Relación tutor-tutorado}

Realizar su propio proceso de búsqueda, por medio de la reflexión sobre su propia práctica, y como resultado de ello, introducir mejoras progresivas en su proceso de enseñanza, era otra gran tarea.

La tutoría, en palabras de Padilla, Bravo, Padilla Muñoz y Castellanos (2004), es entendida como el acompañamiento y apoyo docente de carácter individual, ofrecido a los estudiantes como una actividad más de su formación, y puede ser la palanca que sirva para una transformación cualitativa del proceso educativo en el nivel superior.

Debo reconocer que en esta etapa de tutoría y seguimiento de las prácticas profesionales la participación de cinco catedráticos de la facultad de educación de la Universidad de Murcia permitió apoyar las acciones de mejora y contribuir en el desempeño de todos los participantes en cada colegio, sustancialmente aquí se generó una excelente comunicación y coordinación entre ellos y los tutores mexicanos, quienes hicimos las visitas de seguimiento a las prácticas del estudiantado en cada Colegio o estudiantes que según su especialidad de la licenciatura en educación secundaria, se incorporaron a un instituto denominado Educación Secundaria Obligatoria (ESO).

La práctica docente es una actividad dinámica y reflexiva que comprende los acontecimientos ocurridos en la interacción entre el maestro y los alumnos, es el rol que el profesor juega día con día dentro del aula. En palabras de Fierro, Fortoul y Rosas (1999):

El trabajo del maestro está situado en el punto en que se encuentran el sistema escolar (con una oferta curricular y organizativa determinada), y los grupos sociales particulares. En este sentido, su función es mediar el encuentro entre el proyecto político educativo, estructurado como oferta educativa, y sus destinatarios, en una labor que se realiza cara a cara (p. 85).

En este contexto, las habilidades docentes como conjunto de recursos que permiten al profesor desarrollar la acción de formación trata de favorecer clases con calidad, de tal forma que el estudiantado aprenda los conocimientos significativos y consiga alcanzar los objetivos pedagógicos planteados en las secuencias didácticas del día a día. 

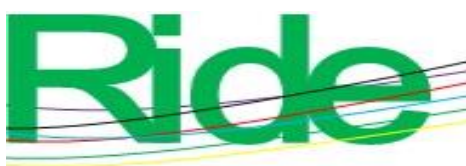

Revista Iberoamericana para la

Investigación y el Desarrollo Educativo

ISSN 2007 - 7467

Un factor inesperado que favoreció la tutoría y permitió de manera natural la visita y el acceso a las aulas para observar y realizar la retroalimentación a cada estudiante fue que dichos colegios forman parte de los escenarios para realizar las prácticas profesionales de quienes estudian las diferentes especialidades en las facultades de la Universidad de Murcia, por ello, todo el profesorado (maestros de vinculación con las prácticas docentes, tutores en cada aula y el mismo personal directivo) tienen como asidua esta terea, en la cual facilitan mucho estos procesos y contribuyen al logro de un espacio de tutoría y de seguimiento efectivo.

\section{Método: desarrollo de habilidades docentes}

Un tema importante dentro del ámbito escolar lo conforman las habilidades que los docentes tutores (docentes de cada grupo de los colegios españoles) emplean para llevar a cabo el proceso de aprendizaje.

Asimismo, la función docente y su contribución para que la evaluación repercuta en mejores prácticas didácticas es vital en estos procesos de tutoría, ya que se favorece la actualización, al tiempo que se mejoran los niveles de desempeño para el trabajo dentro y fuera del salón de clases.

Figura 2. Modelo de trabajo y seguimiento de las prácticas profesionales en Murcia, España

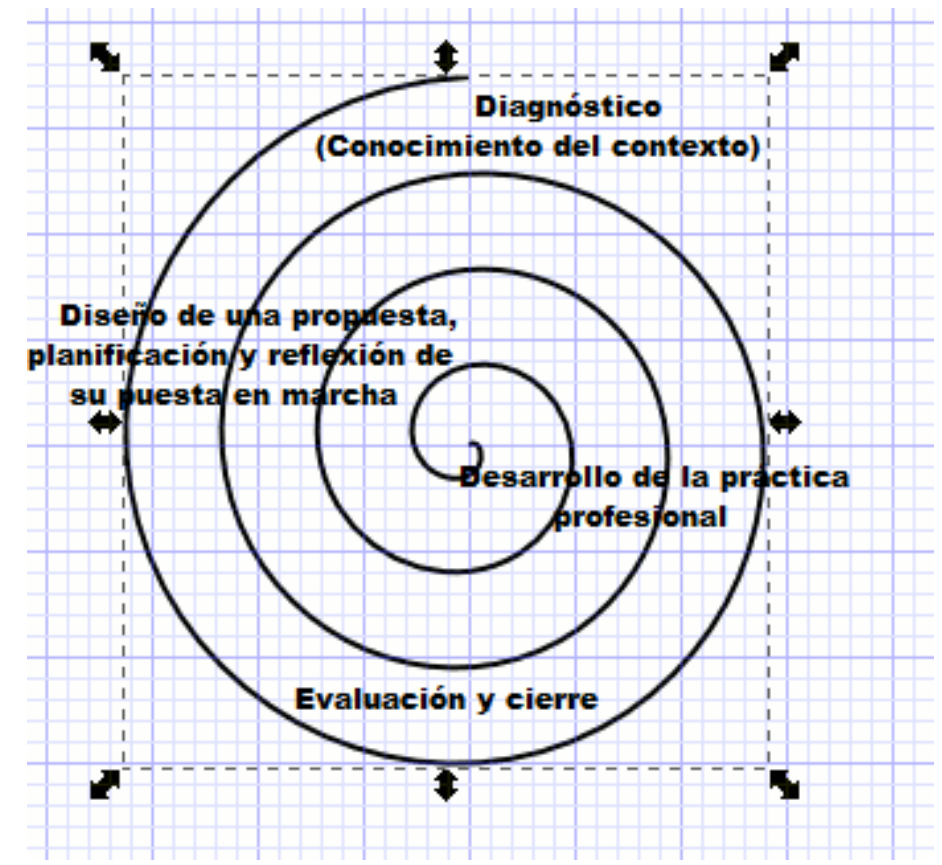

Fuente: autoría propia. 


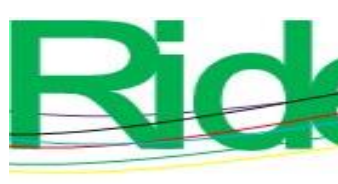

Revista Iberoamericana para la Investigación y el Desarrollo Educativo ISSN 2007 - 7467

Este modelo de trabajo se discutió con el profesorado de la facultad de educación de Murcia, al momento de elaborar el plan de prácticas profesionales, con el argumento de contar con experiencia en el proceso de gestión de las escuelas, incorporación del estudiantado para el conocimiento del contexto escolar y necesidades de su alumnado, desarrollo de sus planeaciones didácticas, seguimiento, evaluación y cierre de las actividades, por lo que accedieron a incorporarlo como modelo de trabajo propio.

Otro aspecto sobresaliente lo constituye el dominio de estrategias didácticas y el manejo de grupos que el estudiantado conocía y aplicaba en contextos mexicanos (Díaz, 2016; Pimienta, 2012); Instituto Tecnológico y de Estudios Superiores de Monterrey [Itesm], 2000), puesto que ello permitiría poner en movimiento dichos saberes en cada colegio, ya que el trabajo por la naturaleza y los tiempos determinados para el momento o etapa del ciclo escolar así lo pretendía. Habría que considerar las fechas, el ciclo escolar estaba casi por concluir, así que era necesario aplicar estrategias y clases que redundaran en lo práctico y significativo, tanto en la docencia, como en la tutoría y la gestión, según las necesidades identificadas a través de dichos procesos y el nivel educativo donde realizaron su práctica profesional.

No existe un modelo único en el marco de la investigación-acción, ya que se trata de adaptar en cada caso específico el proceso de investigación a las condiciones únicas de cada situación concreta (Damiani, 1994). No obstante, para efectos de esta intervención, se precisarán algunas fases o etapas siguiendo un modelo en espiral, en ciclos sucesivos (Carr y Kemmis, 1988), que varían de acuerdo a la complejidad de la problemática.

\section{Resultados: seguimiento de las prácticas profesionales}

Algunos aspectos formativos en los que el estudiantado de escuelas normales mexicanas se destacaron fueron validados mediante un instrumento aplicado; cabe destacar que por razones que escapan al estudio, solo lo contestaron 22 de los 33 participantes y que tiene un carácter informativo, en el que no se pretende generalizar ningún aspecto, sino describir y reflexionar sobre cada proceso, ya que el trabajo se enfoca en reconocer que cada estudiante tuvo que realizar una serie de ajustes metodológicos en función de sus necesidades académicas específicas, ya que en algunos casos los jóvenes pueden presentar problemas para aprender de la misma forma que el resto de sus compañeros; el acercamiento al contexto, su oportunidad de practicar permanentemente en sus aulas, así como el tiempo, materiales distintos de estudio, ayudas en distintas o nuevas técnicas y estrategias de aprendizaje y actividades o dinámicas 
inherentes al propio colegio o el instituto asignado, fueron aspectos que muchas ocasiones estaban fuera de su alcance.

Gran parte del interés de este proceso radica en saber cómo pusieron en movimiento las habilidades docentes aprendidas durante sus prácticas profesionales en un contexto diferente al que comúnmente practicaban, así como identificar si la planeación y las actividades de aprendizaje generan un impacto y ambiente propicio para el estudiante de educación básica de Murcia, España.

Tabla 2. Nivel, especialidad o servicio educativo de procedencia y asignado para el estudiantado en Murcia, España

\begin{tabular}{|c|c|c|}
\hline & $\begin{array}{c}\text { No. } \\
\text { Alumno }\end{array}$ & Catedráticos \\
\hline $\begin{array}{l}\text { Licenciatura en educación } \\
\text { Primaria }\end{array}$ & 10 & 1 \\
\hline $\begin{array}{l}\text { Licenciatura en educación } \\
\text { especial (audición y lenguaje) }\end{array}$ & 3 & \\
\hline $\begin{array}{l}\text { Licenciatura en educación } \\
\text { Preescolar (Infantil) }\end{array}$ & 7 & 1 \\
\hline Licenciatura en educación física & 2 & \\
\hline Especialidad en Ingles & 3 & \\
\hline$\approx$ Lengua (español) & 1 & \\
\hline 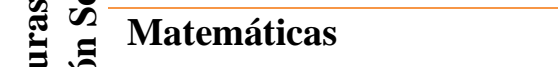 & 3 & \\
\hline Begegía & 2 & \\
\hline 递 & 1 & \\
\hline Historia & 1 & \\
\hline Total & 33 & 2 \\
\hline
\end{tabular}

Fuente: autoría propia. 

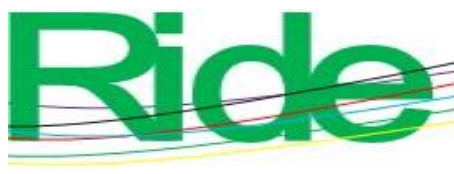

Revista Iberoamericana para la

Investigación y el Desarrollo Educativo

ISSN 2007 - 7467

El estudiantado manifiesta diversos estilos y formas de aprendizaje y ante esto el docente debe jugar un papel muy significativo; se considera vital, en términos de trabajo docente, que el tutor mexicano sea capaz de desarrollar las habilidades y actitudes necesarias para ser guía fundamental en el proceso de enseñanza-aprendizaje al impartir conocimientos teórico-metodológicos dentro y fuera del aula.

Existen elementos relevantes de la participación del estudiantado, como oportunidad de realizar una reflexión respecto al desempeño de sus prácticas profesionales en contextos diferenciados y su nivel de dominio, por los que en los siguientes dos gráficos, sin pretender generalizar, se les preguntó:

- De acuerdo con tu formación académica en la escuela normal de procedencia, ¿en qué nivel de dominio te encuentras para realizar tus prácticas profesionales en el colegio o instituto de práctica en España?

Gráfica 1. Prácticas en otro contexto escolar

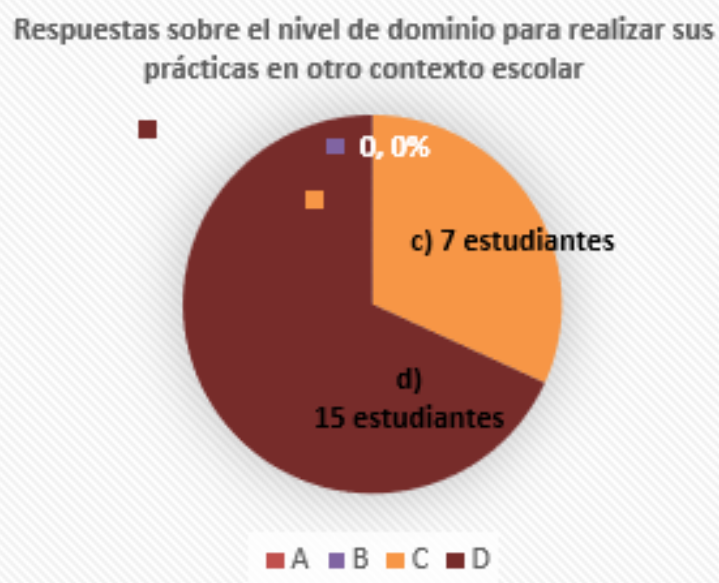

a) con grandes dificultades

b) con algunos retos y limitaciones

c) en buenas condiciones

d) en excelentes condiciones

Fuente: autoría propia.

- Conforme al nivel educativo, modalidad o especialidad con la que te estás formando en la escuela normal de tu procedencia, ¿cómo consideras la formación didáctica que te está ofreciendo para realizar una estancia académica en el colegio o instituto asignado? 


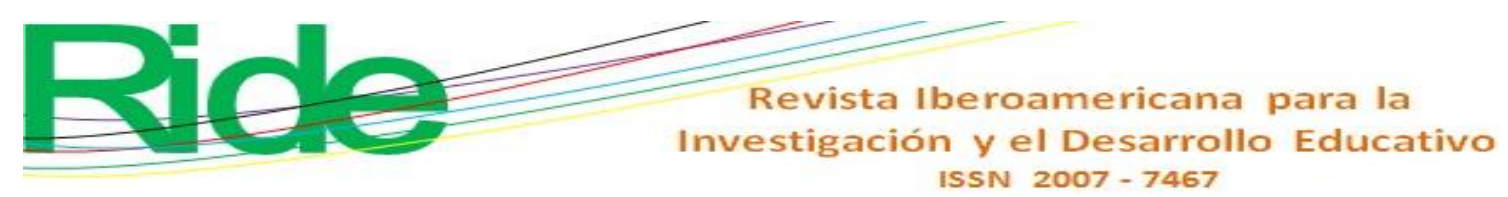

Gráfica 2. Formación didáctica

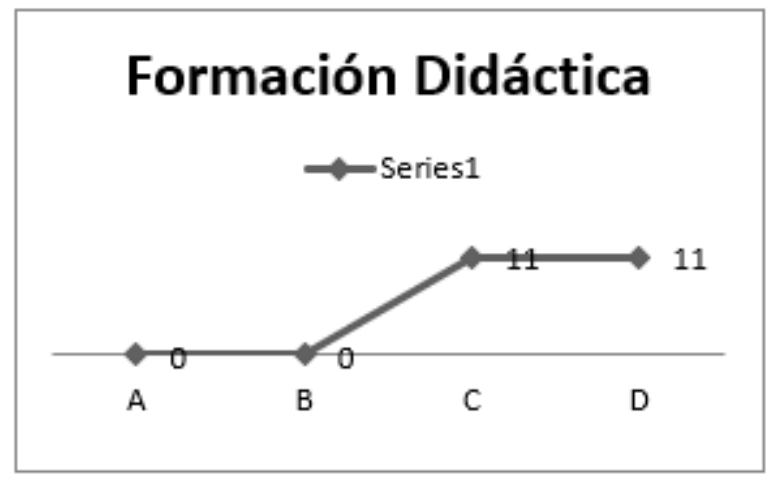
A. Limitada
B. Regular
C. Buena
D. Excelente

Fuente: autoría propia.

Existen, de manera implícita, varias preocupaciones sobre el logro del perfil de egreso y el desarrollo de competencias profesionales, especialmente, ante los momentos coyunturales por lo que atraviesa el sistema educativo mexicano y en particular el de las escuelas normales como formadoras de docentes.

Con los indicadores presentes en estos dos cuestionamientos del instrumento de seguimiento resulta alentador, ya que una parte de la radiografía que presenta el alumnado que contestó este apartado, coloca en los mejores resultados tanto su formación didáctica, como su congruencia en el desarrollo de sus prácticas profesionales.

Algunos indicadores que nos permiten reflexionar sobre los retos y tensiones de este proceso, en el que se observa reciprocidad y hasta cierta corresponsabilidad con el nivel de desempeño esperado durante las prácticas profesionales del estudiantado, lo reflejan los siguientes cuestionamientos presentes en el instrumento, en que se observan bondades reflejadas de este enfoque de investigación-acción, tales como:

a) Identificación de las fuerzas sociales y de las relaciones que están detrás de su experiencia en prácticas anteriores en contextos diferenciados.

b) La generación de nuevos conocimientos al investigador y a los grupos involucrados.

c) La movilización y el reforzamiento de las organizaciones de base.

d) La optimización del empleo de los recursos disponibles, basándose en el análisis crítico de las necesidades. 
e) En algunos casos, después de la comprobación de los resultados en la realidad, cabe la posibilidad de iniciar un ciclo nuevo de la investigación-acción.

- ¿Recibiste asesoría por parte de tu tutor mexicano que apoyara tus prácticas profesionales en el colegio o instituto asignado?

Gráfica 3. Asesoría académica

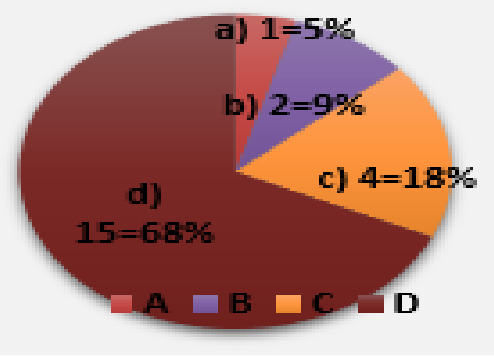
a) Casi nuca
b) Algunas veces
c) Frecuentemente
d) Siempre

Fuente: autoría propia

Gráfica 4. Dificultades de los tutorados

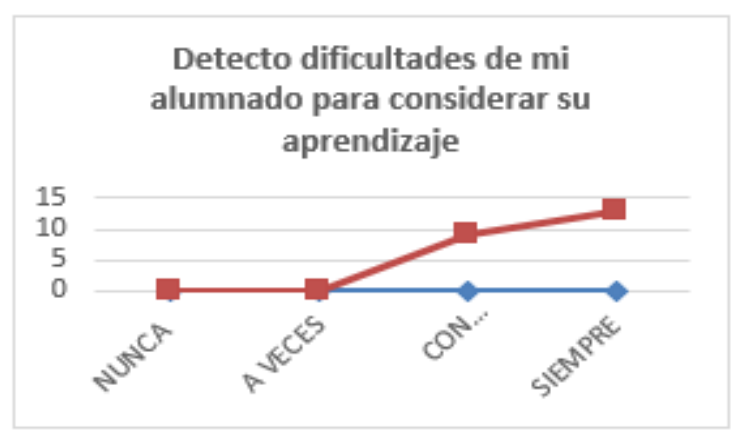

$\begin{array}{lc}\text { Nunca } & 0 \\ \text { A veces } & 0 \\ \text { Con frecuencia } & 9 \\ \text { Siempre } & 13 \\ \text { Total } & 22\end{array}$

Fuente: autoría propia. 


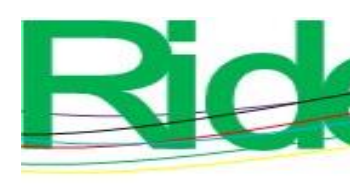

Revista Iberoamericana para la

Investigación y el Desarrollo Educativo

ISSN 2007 - 7467

Las condiciones para brindar la asesoría y seguimiento al estudiantado fueron idóneas, tanto en infraestructura, como en los tiempos, ya que contábamos por parte de la Universidad de Murcia, con un aula especializada en turno vespertino, con proyector, computadora y mesas de trabajo, lo cual permitió la retroalimentación de las actividades realizadas, así como las sugerencias sobre el tipo y cantidad de ayuda pedagógica que brindaban o que solicitaban para las clases de su alumnado.

En lo relacionado a la asesoría de sus prácticas profesionales en cada colegio o instituto, se diseñó un plan de visitas donde el total de alumnos tuviera al menos tres de seguimiento, estableciendo un primer momento destinado para la revisión del diagnóstico y descripción del contexto en el que se involucraron; la segunda visita consistía en observar su trabajo frente al grupo y revisar si las estrategias planeadas en su cuadernillo de práctica correspondía a lo sugerido y, por último, la visita de cierre, en que el estudiantado diseñó una actividad cultural de vinculación México-España donde destacaran actividades culturales, como altares de muertos, rimas de calaveras, exposiciones de la moneda mexicana, presentación de personajes mexicanos famosos, entre otras acciones que resultaron de gran interés para toda la gente involucrada en los colegios y catedráticos de la Universidad de Murcia.

\section{Conclusiones}

Sin duda, un programa o investigación siempre tendrá acciones de mejora; esta experiencia no es la excepción, sin embargo, se debe reconocer que durante las acciones realizadas en los diferentes escenarios de este modelo de trabajo y el seguimiento por parte de todos los involucrados, la madre de todas las habilidades profesionales fue la actitud y la disposición al trabajo cooperativo y de ayuda mutua.

Con base en los apartados del presente artículo, a continuación, se anotan algunas conclusiones.

Sobre la participación colegiada, se presentaron aportaciones entre todos los involucrados en el proceso, caracterizándose por la horizontalidad en la interacción, ya que las aportaciones realizadas al trabajo colegiado fueron consideradas como valiosas; no se trató de un proceso de participación directiva, sino de mantener una comunicación efectiva, de respeto a las diferencias individuales y de asumir un rol de docente que realmente formara parte de ambas comunidades, la del colegio o instituto asignado y la de la facultad de educación de la Universidad de Murcia. 


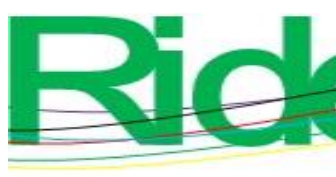

Revista Iberoamericana para la

Investigación y el Desarrollo Educativo

ISSN 2007 - 7467

Sobre la relación tutor-tutorado, la perspectiva del trabajo tutelar quedó atendido en forma muy diversificada, ya que se contó con tres tipos de tutores, el profesor titular del grupo, quien estaba de tiempo completo con el estudiantado y podía sugerir las adecuaciones al trabajo en forma permanente durante su intervención educativa; el tutor de la Universidad de Murcia, que consideraba su participación con el personal directivo y docente de cada colegio, en el caso de quien estuvo asignado a un instituto, se comentaba su desempeño con los responsables del departamento (especialidad o área) de cada licenciatura del estudiantado, y el tutor mexicano, quien interactuaba permanentemente con el estudiantado y logró que fluyera la comunicación académica entre los otros tutores y lograran incorporar a sus prácticas profesionales, tanto las recomendaciones, como los productos de aprendizaje para la toma decisiones en su propia práctica profesional.

Sobre el desarrollo de habilidades docentes, un marco de referencia siempre tiene validez si se relaciona con los niveles de desempeño alcanzado, y para ello, se sintetizará en función de haberse cumplido con el modelo de trabajo y seguimiento de las prácticas profesionales presentadas, en que se destaca el cumplimiento de los objetivos de aprendizaje y metas curriculares; el diseño del plan de trabajo presentando su propuesta de intervención y la evaluación y seguimiento del estudiantado. El seguimiento de las prácticas profesionales fue verificado tanto en los registros, como en las visitas de seguimiento en cada colegio, con 100\% de asistencia y cumplimiento del estudiantado, ni siquiera por enfermedad o accidente un alumno faltó en la entrega de sus planeaciones didácticas o de su plan de acción y evaluación, por lo que haber cumplido en horarios, fechas y con las cuatro etapas del proceso determinado para este modelo de participación, corrobora en mucho el éxito y motivación hacia todo el proceso.

Las autoridades educativas de los propios colegios y los tutores de la universidad comentaron que la estancia superaba en mucho las expectativas que tenían de la experiencia académica; además, el estudiantado para los tutores mexicanos también era nuevo, puesto que solo dos estudiantes eran de Veracruz, México, y nunca habían sido alumnos en la Escuela Normal.

Estas reflexiones refieren a esfuerzos intelectuales de una experiencia exitosa vinculada a una estancia académica con alumnos normalistas en el extranjero, y se debe señalar que se espera que quienes estén o deseen transitar por situaciones semejantes, consideren estas preocupaciones para mejorar las condiciones de la experiencia académica. 


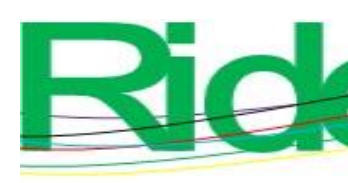

Revista Iberoamericana para la

Investigación y el Desarrollo Educativo

ISSN $2007-7467$

\section{Referencias}

Alvarez-Gayou, J. (2003). Cómo hacer investigación cualitativa. Fundamentos y metodología. México: Paidós

Carr, W. y Stephen, K. (1988). Teoría crítica de la enseñanza. La investigación-acción en la formación del profesorado. Barcelona, España: Martínez Roca.

Damiani, L. (1994). La diversidad Metodológica en la sociología. Caracas, Venezuela: Tropykos.

Díaz Barriga, F. et al. (2016) Estrategias docentes para un aprendizaje significativo. Una interpretación constructivista. México: Mc Graw Hill.

Fierro, C. y Rosas, L. (1999). Transformando la práctica docente: una propuesta basada en la investigación-acción. México: Paidós.

Instituto Tecnológico y de Estudios Superiores de Monterrey [Itesm]. (2000). Las técnicas didácticas en el modelo educativo del Tec de Monterrey. México: Itesm.

Lewin, K. (1946). Action reseach and minority issues. United States: Journal of social.

Moser. (1978). Critical qualitive research methods. London, England: Sage Publications.

Padilla, L., Bravo P., Padilla, M. y Castellanos, R. (2004). Programa Institucional de Tutoría Academica. La tutoría Académica y la calidad de la educación. México.

Pelayo, P. M. (2012) Capital social y competencias profesionales: Factores condicionantes para la inserción laboral. México: Eumed. Recuperado de http://www.eumed.net/.

Pimienta, P. J. (2012). Estrategias de enseñanza-aprendizaje. Docencia universitaria basada en competencias. México: Pearson.

Sandín, E. (2003). Investigación Cualitativa en Educación: Fundamentos y Tradiciones. Madrid, España: McGraw Hill.

Secretaría de Educación Pública [Sep]. (1997). Programa de Seminario de trabajo docente II. Octavo Semestre. Licenciatura en Educación primaria. México.

Secretaría de Educación Pública [Sep]. (1999). Programa de Seminario de trabajo docente II. Octavo Semestre. Licenciatura en Educación preescolar. México.

Secretaría de Educación Pública [Sep]. (2012). Plan de estudios. Práctica profesional. Séptimo y octavo Semestres. Licenciatura en Educación primaria. México.

Zarzar Charur, C. (2009). Habilidades básicas para la docencia. México: PATRIA. Recuperado de https://caricaturahistorica.files.wordpress.com/2013/12/habilidades-basicas-para-ladocencia.pdf. 